\title{
Treatment of bilateral ankylosis of the temporomandibular joint with temporalis superficial fascia interpositional flap
}

\author{
Harmas Yazid Yusuf, Alwin Kasim, Tis Karasutisna \\ Department of Oral and Maxillofacial Surgery Faculty of Dentistry Universitas Padjadjaran \\ Hasan Sadikin Hospital-Bandung, West Java, Indonesia
}

\begin{abstract}
Ankylosis of the temporomandibular joint (TMJ) involves fusion of the mandibular condyle to the base of the skull. Trauma and infection are the leading causes of ankylosis. A case of true bilateral ankylosis of the temporomandibular joint is presented. A 21 year old male patient had a multiple bone fractures history at the age of 13 due to sports injury. A TMJ injury might not be detected at that time resulting in a progressive restriction of his mouth opening. He presented with almost complete lack of mobility of the mandible. Surgical treatment was a resection of the ankylotic bone mass, interposition temporalis superficialis fascia flaps, and early mobilization and aggressive physiotherapy. The functional results showed good remarks.
\end{abstract}

Key words: Ankylosis, bilateral ankylosis, temporomandibular joint, interpositional flap

\section{INTRODUCTION}

Ankylosis (the word comes from the Greek, ankulos meaning crooked or bent) is defined as loss of joint movement resulting from fusion of bones within the joint or calcification of the ligament around it. ${ }^{1}$

Ankylosis of the temporomandibular joint (TMJ) involves fusion of the mandibular condyle to the base of the skull. When it occurs in a child, it can have devastating effects on the future growth and development of the jaws and teeth. Furthermore, in many cases it has a profoundly negative influence on the psychosocial development of the patient, because of the obvious facial deformity, which worsens with growth. ${ }^{2,3}$

Different pathologies can originate mandibular ankylosis that affect the temporomandibular articulation as to the surrounding structures. The most commonly associated causes are traumatic, local or distant infections and the systemic illnesses.

The first sign of a significant problem may be increasing limitation of jaw opening, usually noticed by the dentist. Pain is uncommon. However, in a young patient, a joint injury may not be noticed immediately. Early diagnosis and treatment are crucial if the worst sequelae of this condition are to be avoided. ${ }^{2,3,4}$

In this report, we describe the use of temporalis superficial fascia interpositional flap to treat bilateral ankylosis of TMJ.

\section{CASE REPORT}

A 21-year-old male came to the Department of Oral and Maxillofacial Surgery Faculty of Dentistry Padjadjaran University Sadikin or Hasan

Correspondence author: Harmas Y, Department of Oral and Maxillofacial Surgery Faculty of Dentistry Universitas Padjadjaran Hasan Sadikin Hospital-Bandung Jl.Pasteur 38 Bandung,West Java-Indonesia, Tel./Fax.+6222-2041196/2036169 
Figure 1. Pre-treatment interincisal-measurement oral opening was only $3 \mathrm{~mm}$ with poor oral hygiene

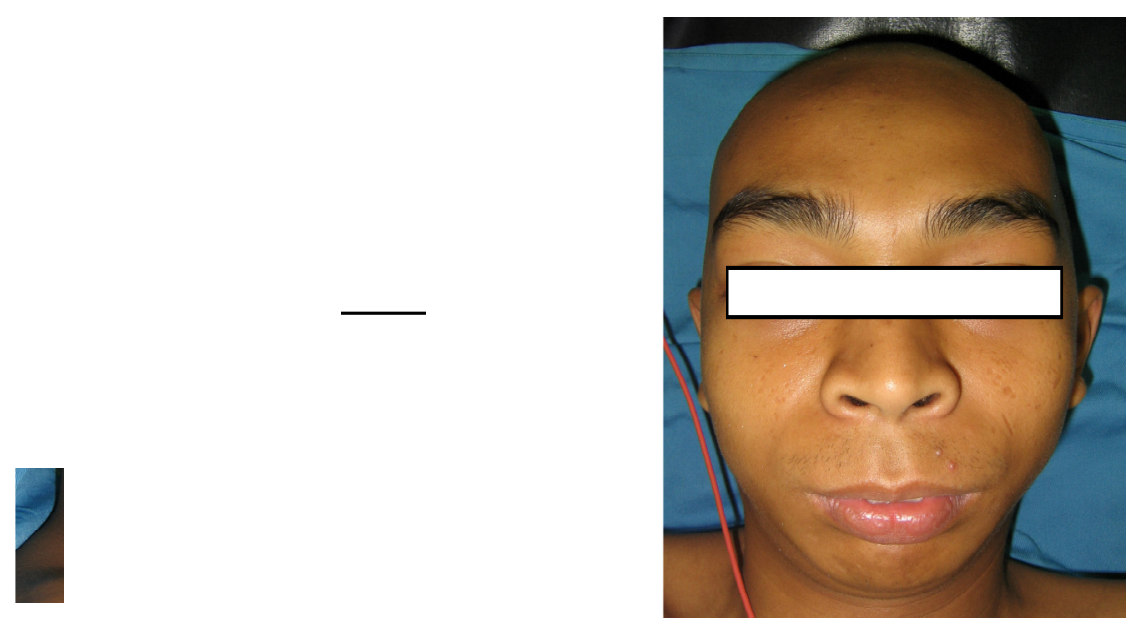

Figure 2. Mandibular retrognathia
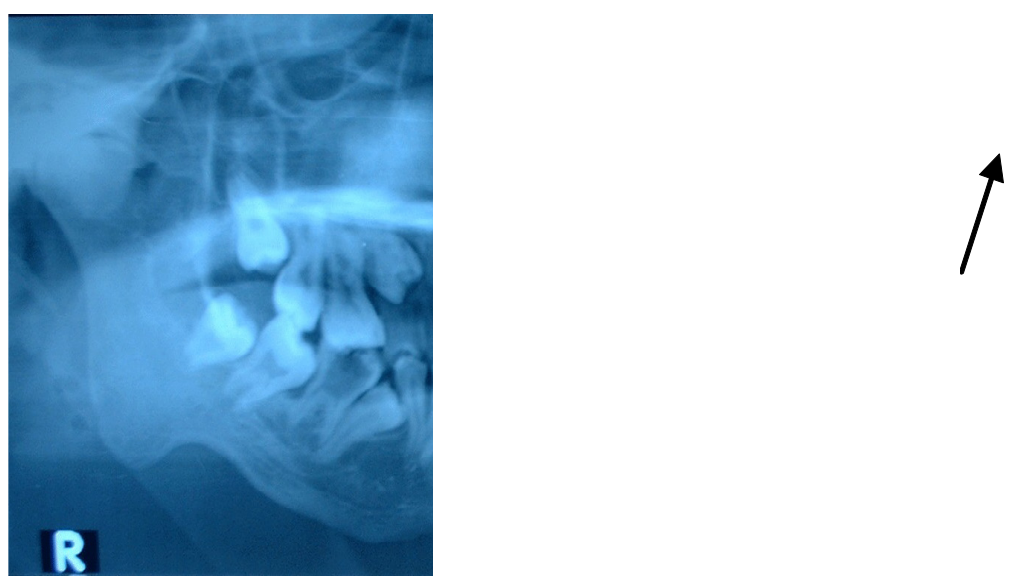

Figure 3. Panoramic radiography showed bilateral TMJ ankylosis (arrow).

Sadikin Hospital, Bandung, West Java, Indonesia with chief complain could not open his mouth widely started at the age of 13 due to sport injury. He had also a history of multiple bone fractures i.e. bilateral humerus and left tibia that have already been corrected by orthopedic surgeons. A TMJ injury might not be detected at that time. Oral examination revealed poor oral hygiene, difficulty to pronounce and masticate and had only liquid nutrition. Interincisal-measurement oral opening was only $3 \mathrm{~mm}$ (Fig. 1) with mandibular retrognathia (Fig. 2).

Panoramic radiography examination was positive for old bilateral mandibular condylar fractures and lack of interarticular space and disturbances in mandibular growth (Fig. 3). 

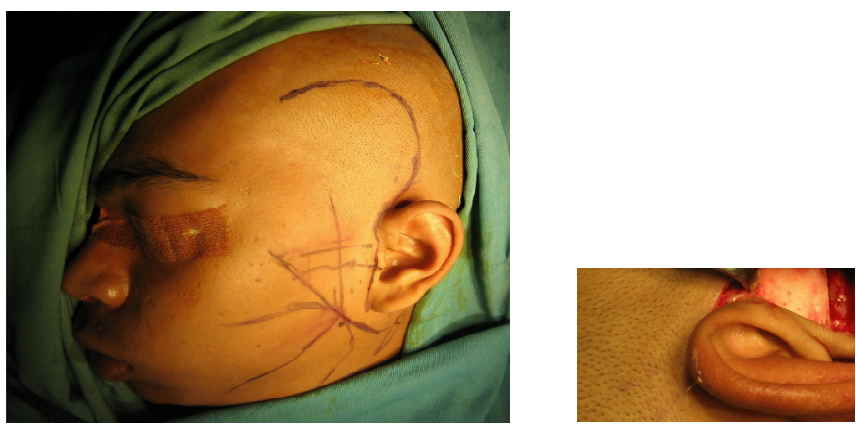

Figure 3. Al Kayat - Bramley TMJ approach (A) and osteartrectomy (B)
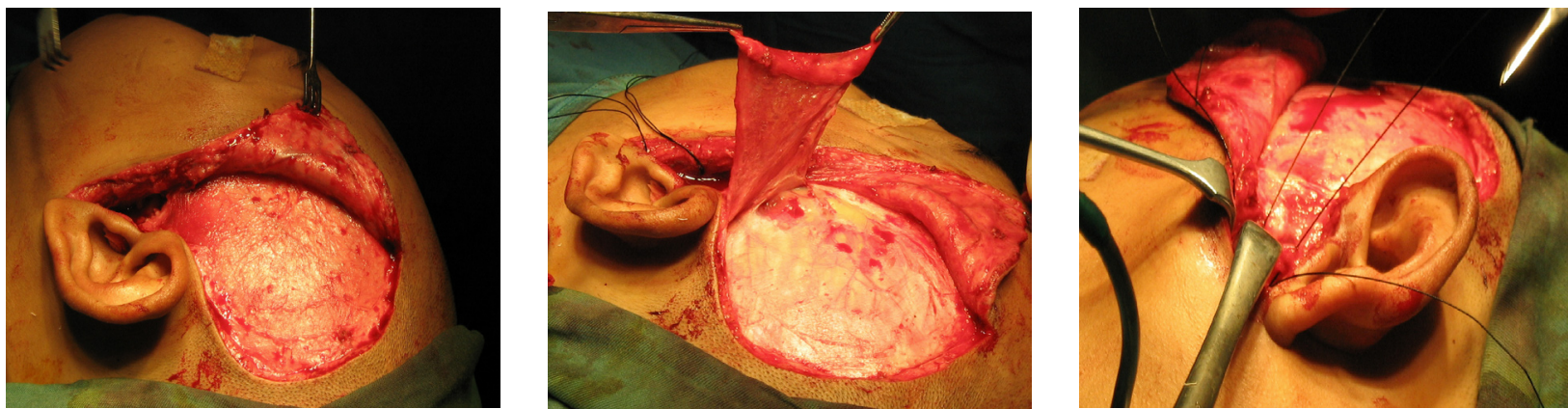

Figure 4. Interposition temporalis superficial fascia flap procedure. A. Incision design. B. The temporalis superficial fascia flap. C. Interposition of the fascia between the bony segments

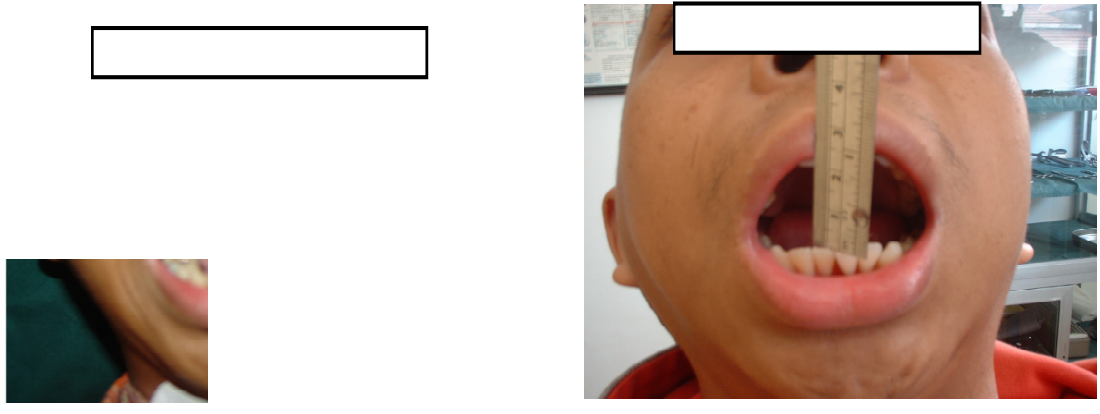

Figure 5. Post treatment interincisal-measurement oral opening $25 \mathrm{~mm}$ in one week (A) and $30 \mathrm{~mm}$ in the next two months (B).

A of bilateral TMJ Ankylosis with vertical maxillary excess, malocclusion dentition and mandibular retrognathia was made. The treatment plan was bilateral TMJ gap arthroplasty with interposition of temporalis superficial fascia flap, early mobilization and physiotherapy, future orthodontic treatment, and future orthognathic surgery.

The patient underwent an operation using general anesthesia with prophylactic antibiotic cover. Tracheotomy was done before the TMJ operation and an Al Kayat-Bramley TMJ approach was used (Fig. 3). An aggressive $1 \mathrm{~cm}$ osteoartrectomy was performed to both condyles and the temporalis superficial fascia interpositional flap was inserted between the bony segments (Fig. 4).

The postoperative course was uneventful. The patient presented interincisal-measurement oral opening $25 \mathrm{~mm}$ in one week and $30 \mathrm{~mm}$ in the next two months post operative (Fig. 5). Ongoing long-term follow up continues.

\section{DISCUSSION}

Mandibular hypomobility resulting from TMJ ankylosis is classified according to location (intracapsular or extracapsular), type of tissue involved (bony, fibrous or fibro-osseous) and extent of fusion (complete or incomplete). The causes 
and treatment of TMJ ankylosis have been well documented, with trauma and infection identified as the two leading causes. If the cause is trauma, it is hypothesized that intra-articular hematoma, along with scarring and formation of excessive bone, leads to the hypomobility. ${ }^{5}$

Infection of the TMJ most commonly occurs secondary to contiguous spread from otitis media or mastoiditis, but it may also result from hematogenous spread of infectious conditions such as tuberculosis, gonorrhea or scarlet fever. Systemic causes of TMJ ankylosis include ankylosing spondylitis, rheumatoid arthritis and psoriasis. ${ }^{4}$

In children, TMJ ankylosis can result in mandibular retrognathism with attendant esthetic and functional deficits. These problems have functional and esthetic implications, as well as causing difficulties pertaining to nutrition and oral hygiene. Therefore, treatment should be initiated as soon as the condition is recognized, with the main objective of re-establishing joint function and harmonious jaw function. Surgical options for treatment include gap arthroplasty which is still preferred and interposition surgery which is the standard procedure. Satisfactory surgical correction of temporomandibular joint ankylosis (TMA) is limited by a high recurrence rate, particularly in patients who underwent surgery without use of interposition material. , $, 5,6$

Interposition materials used included temporalis fascia, temporalis muscle, silastic sheet and cartilage (costochondral) grafts, etc. Simple gap arthroplasty appears to be of limited value in TMA surgery, particularly due to the high risk of recurrent joint ankylosis. Silastic sheet interposition carries the risk of infection and extrusion in the long term.

Costochondral graft is satisfactory in the young age group ( $<12$ years) as it provides a growth centre for condylar growth; however two surgical sites are required which can be a source of parental refusal for operation. ${ }^{2}$

The temporalis superficial fascia flap is an autogenous graft that has the advantages of close proximity to the TMJ, minimal surgical morbidity, and successful clinical results. Among the various surgical options available for treating TMA, use of interposition surgery with temporalis fascia and/ or muscle provides the most satisfactory results for all age groups. ${ }^{3,5,6}$

This case demonstrated that use of a temporalis superficial fascia as graft arthroplasty in bilateral TMJ ankylosis showed a significant improvement in the superior inferior position of the mandible. The functional result showed good remarks.

\section{CONCLUSION}

The temporalis superficial fascia flap is an autogenous graft that has the advantages of close proximity to the TMJ, minimal surgical morbidity, and successful clinical results. Use of a temporalis superficial fascia as graft arthroplasty in bilateral TMJ ankylosis showed a significant improvement in the superior inferior position of the mandible. The functional result showed good remarks.

\section{REFERENCES}

1. The Merck manual-home edition. Disorders of the temporomandibular joint. htm.

2. Abbas I, Jamil $M$, Jehanzeb $M$, Ghous SH. Temporomandibular joint ankylosis: experience with interpositional gap arthroplasty at Ayub Medical College Abbottabad. Ayub Med Coll Abbottabad 2005;17(4).

3. Su-Gwan K. Treatment of temporomandibular joint ankylosis with temporalis muscle and fascia flap. Int J Oral Maxillofac Surg 2001;30(3):189-93.

4. Garcia-Aparicio L, Parri EJ, Sancho MA, et al. Temporomandibular joint ankylosis (TMA) in children. Cir Pediatr 2000;13(2):62-3.

5. Valentini V, Vetrano $S$, Agrillo $A$, et al. Surgical treatment of TMJ ankylosis: our experience (60 cases). J Craniofac Surg 2002;13(1):59-67.

6. Balaji SM. Modified temporalis anchorage in craniomandibular reankylosis. Int J Oral Maxillofac Surg 2003;32(5):480-5. 